\title{
Anniversary
}

\section{CP violation: past, present and future}

\section{Highlights from a meeting to celebrate the 50th anniversary of a surprising discovery that continues to tantalize.}

Fifty years after the seminal discovery of CP violation by James Christenson, James Cronin, Val Fitch and René Turlay, Queen Mary University of London held a meeting on 10-11 July to celebrate the anniversary. This stimulating retrospective was attended by around 80 participants, many of whom had been involved in the numerous experimental and theoretical developments in CPviolation physics during the intervening half-century. The primary focus was to review the experimental and phenomenological aspects of CP violation during the past 50 years, but the meeting also included talks on the future of CP-violation experiments with heavy flavours as well as with neutrinos.

The meeting got off to a barnstorming start with talks by Nobel prize laureates Jim Cronin (1980) and Makoto Kobayashi (2008). Cronin explained that since René Turlay had sadly passed away in 2002, while Val Fitch was no longer able to travel and contact with Jim Christenson appeared to have been lost, he alone of the original team was available to attend such meetings. He carefully outlined the historical context in neutral-kaon physics surrounding the discovery of CP violation at Brookhaven in 1964, giving significant credit to Robert Adair, whose earlier experiment had discovered "anomalous regeneration of $\mathrm{K}_{\mathrm{L}}^{0}$ mesons" in 1963. This in turn had stimulated Fitch to suggest to Cronin that the latter's existing apparatus might be used to repeat and improve upon that measurement with 10 times the sensitivity. A search for $\mathrm{CP}$ violation in $\mathrm{K}^{0}$ decays to two charged pions would be an additional test that could be made as a by-product of the new experiment.

The proposal was made in 1963 and the experiment commenced within three weeks (CERN Courier July/August 2014 p21). Illustrating his talk with photographs of the original laboratory notebooks kept by the team, Cronin explained that it was Turlay alone who performed the analysis for the CP-violation signal, and found a signal corresponding to 40 two-pion $\mathrm{K}_{\mathrm{L}}^{0}$ decays by Christmas 1963. This result implied that CP violation was manifest in the neutral-kaon system, corresponding, for example, to an admixture of the $\mathrm{CP}=+1$ component in the long-lived $\mathrm{K}^{0}$ at the level of $2.3 \times 10^{-3}$ - a result later confirmed by other experiments.

Cronin continued by reviewing the later experimental work in

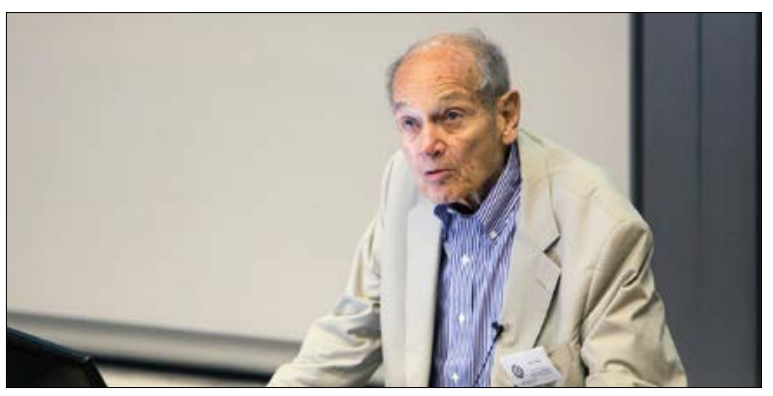

Jim Cronin opened the meeting, 50 years after the famous paper was published.(Image credits: all photos Honor-Clare Elliot.)

CP-violation physics with neutral kaons, confirming and building upon the original discovery, and culminating in the unequivocal demonstration, almost 40 years later, of direct $C P$ violation in the kaon system (CERN Courier July/August 2014 p23). His talk stimulated several questions. One participant commented that the time from submission of the seminal paper to publication was very short. Another asked if there had been any expectation or indication of a CP-violation signal before the experiment. Cronin responded in the negative: "We did not even think $\mathrm{CP}$ violation was the most important thing - we really wanted to measure $\mathrm{K}_{\mathrm{S}}^{0}$ regeneration." A former student of Cronin commented that at the time he was "having lectures from these guys", and that he "could tell that something exciting was going on behind the scenes".

\section{Towards a theory}

The second talk was by Kobayashi, who together with Toshihide Maskawa had shown in 1973 how to accommodate CP violation into the gauge theory of electroweak interactions, albeit necessitating their bold suggestion of a third family of quarks - insight for which they were to receive the Nobel prize in 2008. Kobayashi carefully outlined the context in which his decisive work with Maskawa on $\mathrm{CP}$ violation was performed. He had entered graduate school in 1970 at Nagoya, where the theoretical physics group was led by Shoichi Sakata, and where Maskawa had completed his PhD in 1967. Kobayashi explained how their theoretical ideas had been influenced deeply by Sakata's work, especially by his 1956 model of hadrons. This was a forerunner to the quark model that, in particular, stimulated the study of the SU(3) group in the context of particle physics. Moreover, a paper by Sakata together with Ziro Maki and Masami Nakagawa in 1962 had included a theory describing mixing 
in the lepton sector using a $2 \times 2$ matrix with a single mixing angle.

Maskawa had moved to Kyoto in 1970 and Kobayashi followed him there in 1972, at which point they started to work together on trying to incorporate $\mathrm{CP}$ violation into the recently formulated gauge theory of electroweak interactions. They quickly realized that it would not be possible to achieve this goal with only four quarks, and concluded that extra particles would be needed. Their paper enumerated several possibilities, including the six-quark model with their $3 \times 3$ mixing matrix, which would turn out to be correct. This work, as Kobayshi pointed out, "only took a couple of months".

Two talks followed on the experimental search for CP-violating phenomena with neutral kaons - past and future - by Marco Sozzi of the University of Pisa and Taku Yamanaka of Osaka University. The search for direct $\mathrm{CP}$ violation had needed measurements of $\mathrm{K}_{\mathrm{L}}^{0}$ decaying to two $\pi^{0} \mathrm{~s}$. This was dubbed the "decay where nothing goes in and nothing comes out", but successive experiments succeeded in studying it with staged experimental innovations. Between the first observation of CP violation and the eventual demonstration of direct CP violation in neutral kaons, the number of $\mathrm{K}^{0}$ decays observed increased by $5-6$ orders of magnitude as a result of technological innovations. Much was made of the long drawn-out history of measurements of $\operatorname{Re}\left(\varepsilon^{\prime} / \varepsilon\right)$ - the observable manifestation of direct $\mathrm{CP}$ violation in neutral kaons - with apparent fluctuations (albeit within experimental uncertainties) in its value throughout two generations of experiments on both sides of the Atlantic, before it settled down eventually to its current value of $(1.65 \pm 0.26) \times 10^{-3}$. One participant asked what value of $\eta$ - Wolfenstein's CP-violating imaginary parameter in the Cabibbo-Kobayashi-Maskawa (CKM) matrix - does the measured value of $\varepsilon^{\prime}$ correspond to? Sozzi responded that the cancellations in the calculation of $\varepsilon^{\prime}$ in terms of $\eta$ are so complete that it is not possible to make such a one-to-one correspondence.

In considering the legacy of the neutral-kaon experiments, Cronin commented that although a great deal of work had been done during the years to measure the values of the elements of the CKM matrix, it was still a great mystery as to why their values are what they are, and he asked whether theory had left the field "in trouble" over this. However, Yamanaka could "only share his frustration". The baton for CP-violation experiments with kaons now passes to the K0TO ( $\mathrm{K}^{0}$ to Tokai) experiment at the Japan Proton Accelerator Research Complex (J-PARC), and the NA62 experiment at CERN.

The meeting moved on next to the $\mathrm{B}$ factories, with two historical talks by Jonathan Dorfan, now of the Okinawa Institute of Science and Technology, and Masanori Yamauchi of KEK, respectively, on the PEP-II storage rings at SLAC and the KEK-B collider. The large mixing among neutral $\mathrm{B}$ mesons and their relatively long lifetimes offered the possibility to observe large $\mathrm{CP}$ violation in their decays, but it was necessary to produce them in motion to allow their decay times to be resolved. The large cross-section in the region of the $\mathrm{Y}(4 \mathrm{~S})$ made it the ideal production environment, but symmetric collisions would have implied near-stationary B mesons. Pier Oddone, together with Ikaros Bigi and

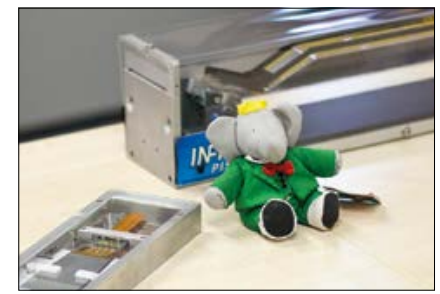

Even BaBar came along to take part in the exhibition.

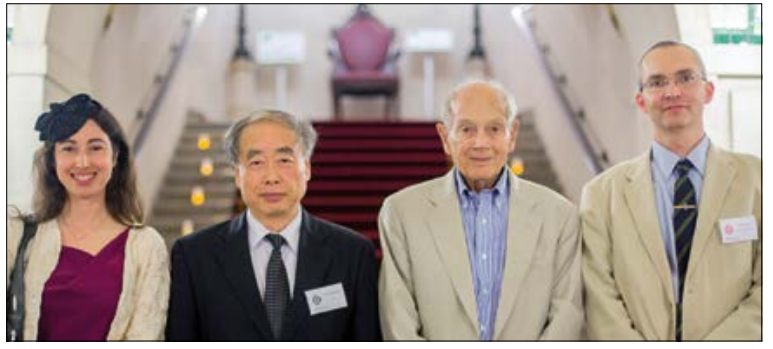

Nobel laureates Makoto Kobayashi, second from left, and Jim Cronin, with the organizers from QMUL, Adrian Bevan, right, and Marcella Bona.

Tony Sanda, proposed a solution in 1987 by suggesting the production of boosted neutral $\mathrm{B}$ mesons using asymmetric pairs of $\mathrm{e}^{+}$and $\mathrm{e}^{-}$beams tuned to the $\mathrm{Y}(4 \mathrm{~S})$ resonance. This approach has been vindicated by the success of the $\mathrm{B}$ factories in comparison with competing ideas, such as fixed-target production by a hadronic beam, for example, at the HERA-B project.

These talks thoroughly reviewed many interesting details of the beam designs. PEP-II and KEK-B pioneered true "factory running" of colliders, with continuous injection used for the first time in these projects. In the end, PEP-II produced a total integrated luminosity of $557 \mathrm{fb}^{-1}$ between 1999 and 2008 , and KEK-B produced $1000 \mathrm{fb}^{-1}$ by its shutdown in 2010. PEP-II was built by an innovative collaboration between the Lawrence Berkeley Laboratory, the Lawrence Livermore National Laboratory, and SLAC. Asked if this was a model for the future, Dorfan replied: "The time was right. The [US Department of Energy] let us manage ourselves. There was no messing with our budget by Congress, which was a great advantage. Physicists were very involved. It couldn't be done now!"

\section{BaBar and Belle}

Next came talks on the experiments at the B factories, BaBar and Belle, in which their histories were given a thorough airing. The BaBar collaboration had asked Laurent de Brunhoff for permission to use the name and image of his father's famous fictional elephant, which was duly given with certain conditions attached. (For example, the elephant can be shown holding something only if he is using his trunk, not his hands or feet.) The collaboration went on to pioneer the technique of blind analysis - not as the first experiment to exploit it, but the first to make it standard throughout its analyses. As David Hitlin of the California Institute of Technology, the first spokesperson of BaBar, recalled in his talk, one collaborator had insisted early on that "we don't need a blind analysis because we know the answer already," which had convinced Hitlin of the need for it.

The presentations gave a virtual tour of BaBar's and Belle's CP-violating and T-violating measurements with $\mathrm{B}$ mesons, probes of new physics, tests of penguin amplitudes, neutral-meson mixing with charm, and tests of CP violation in tau decays. Both experiments proved spectacularly that the CKM $\triangleright$ 


\section{Anniversary}

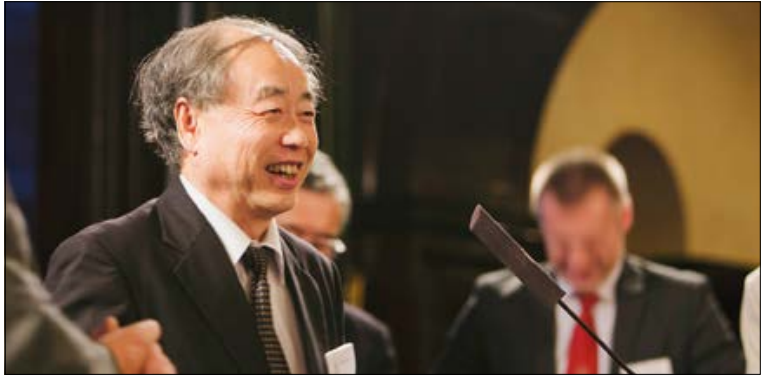

Kobayshi speaks after accepting the book Physics at the $\mathrm{B}$ Factories at the conference dinner.

description of CP violation in the Standard Model is correct. In question time, one collaboration member reported a conversation with a journalist at a conference in Tokyo in 2000. "What's it like to do a blind analysis? - It's the scariest thing I've ever done in my life," had been the candid response. The meeting then turned its attention to the Tevatron at Fermilab, where precise measurements of $B_{s}$ oscillations and related observables gave valuable new constraints on the unitarity triangle, and again provided further detailed confirmation of the Standard Model.

Gilad Perez of the Weizmann Institute then gave a theoretical talk outlining how the physics of the top quark could offer new insights into the flavour problem in the future, especially at the LHC, with unique opportunities for flavour-tagging in top decays. The extremely large mass of the top quark makes it the only quark to decay before it forms hadrons, and this gives unique access in hadron physics to a decaying quark's spin, charge and flavour. Another important effect of the top's large mass is its importance for fine tuning the weak vacuum - had its mass been a mere $3 \%$ greater, the weak vacuum would have been unstable and there would have been no weak interaction in the form observed. The ATLAS and CMS experiments at the LHC have already collected more than five million ti pairs, with many more to come. Semi-leptonic decays of t quarks provide a strong flavour-tagging of the resulting b quarks, making such decays akin to a new type of B factory, barely explored so far.

In an historical overview of the LHCb experiment's genesis, the first spokesperson, Tatsuya Nakada, now of the École polytechnique fédérale de Lausanne, described how it was born out of the "shotgun marriage" of the three earlier proposals for B physics at the LHC: COBEX - a collider-mode forward-spectrometer concept to exploit the large $b \bar{b}$ cross-section in high-energy protonproton collisions; LHB - using a bent crystal for extraction of the beam halo for a fixed-target B experiment; and GAJET - using the gas-jet target concept. The LHC Committee had reviewed the three ideas, and in its wisdom stipulated that there should be a collider-mode experiment, but redesigned under new management to allow the three proto-collaborations to merge into a single entity, which became LHCb. "The first time I think a committee was really clever," Nakada commented. Approval was not trivial, but the impressive results to date have already vindicated the approach taken. A second talk on LHCb by Steve Playfer of Edinburgh University gave a detailed review of its physics output, where the cleanliness of the signatures has surprised even the participants.
$\mathrm{CP}$ violation in B-baryon decays is a promise for the future.

There were also presentations on the contributions to $\mathrm{CP}$ violation physics from ATLAS and CMS at the LHC. These experiments cannot measure $\mathrm{CP}$ violation in purely hadronic $\mathrm{B}$ decays because they do not have the required particle identification to reconstruct the exclusive final states. However, with the huge crosssections available at these energies and the experiments' good lepton-identification capabilities, they are well placed to surpass the $\mathrm{B}$ factories in sensitivity to CP violation in final states in which $\mathrm{J} / \psi$ particles decay to leptons.

Further talks reviewed the theoretical and experimental status of $\mathrm{CP}$ violation in charm and the prospects for its discovery, as well as future prospects at the planned upgrades to both Belle and $\mathrm{LHCb}$, and also at neutrino facilities. The discovery of $\mathrm{CP}$ violation in neutrinos would be the crowning achievement of neutrino-oscillation studies. There were also two detailed reviews of the history of $\mathrm{T}$ violation, first in kaon physics and then in B decays.

A final talk by Marco Ciuchini of INFN/Roma Tre University reviewed the theoretical implications and future perspectives on $\mathrm{CP}$ violation. Again, Cronin wondered why the community is not yet in a position to understand the spectra of fermion masses and mixings, including $\mathrm{CP}$ violation. The speaker responded that "this is the hardest problem". One questioner asked if a deviation from the Standard Model were to be observed with the upgraded LHCb or Belle II, thereby indicating some new physics in virtual-loop processes, what energy machine would be needed to observe such physics directly? The answer, said Ciuchini, would depend on the details of the new physics.

The conference dinner took place at the Law Society in the City of London, in grand surroundings appropriate for a 50th anniversary. During the past six years, BaBar and Belle have been collaborating on a grand review of Physics at the B Factories, and the occasion was used to announce the completion of this monumental tome. It was also a fitting opportunity to present complimentary copies to Cronin and Kobayashi, in honour of their personal contributions to the current understanding of $\mathrm{CP}$ violation.

- For more details on all of the speakers and presentations at the symposium, visit http://pprc.qmul.ac.uk/ research/50-years-cp-violation.

\section{Résumé}

\section{La violation de CP : passé, présent et avenir}

Pour célébrer le cinquantenaire de la découverte décisive de la violation de CP, l'Université Queen Mary de Londres a accueilli les 10 et 11 juillet derniers une réunion spéciale. Cet événement a rassemblé quelque 80 participants, qui étaient nombreux à avoir contribué aux développements expérimentaux et théoriques de la physique de la violation de CP au cours de ce demi-siècle. Si la rencontre visait essentiellement à passer en revue les aspects expérimentaux et phénoménologiques de la violation de CP durant ces 50 dernières années, les participants ont pu également entendre des exposés sur les futurs projets d'expériences de violation de CP avec les saveurs lourdes et les neutrinos.

Paul Harrison, University of Warwick. 